\title{
Modelling the hydromechanical behaviour of expansive granular mixtures upon hydration
}

\author{
Benjamin Darde ${ }^{1,2, *}$, Anh Minh Tang ${ }^{1}$, Jean-Michel Pereira ${ }^{1}$, Patrick Dangla ${ }^{1}$, Jean-Noël Roux ${ }^{1}$, Jean Talandier ${ }^{2}$ and Minh Ngoc Vu ${ }^{2}$ \\ ${ }^{1}$ Laboratoire Navier, UMR 8205 (École des Ponts ParisTech - IFSTTAR - CNRS), France \\ ${ }^{2}$ French national radioactive waste management agency (Andra), France
}

\begin{abstract}
Bentonite pellet-powder mixtures are candidate sealing materials in radioactive waste disposal concepts. The mixture is installed in galleries in dry state as a granular material. The material is progressively hydrated by the pore water of the host rock and becomes homogeneous. Before homogenisation, the granular structure controls the material behaviour. In the present work, a modelling approach able to address particular features of pellet-powder mixtures is introduced. Two domains are considered: i) granular, and ii) homogeneous. The material behaviour before homogenisation is studied through Discrete Element Method (DEM) simulations. Constitutive laws for the granular state are proposed from DEM results. The behaviour of the homogenised material is described by a modified Barcelona Basic Model (BBM). Transition from granular to homogeneous states depends on suction and relative volume fractions of pellets and powder. Swelling pressure tests performed in the laboratory are satisfactorily simulated using this approach.
\end{abstract}

\section{Introduction}

Bentonite pellet-powder mixtures are considered as candidate sealing materials in radioactive waste disposal concepts [1-5]. These materials are characterised by a low permeability, good radionuclide retention capacity, and ability to swell upon hydration.

Pellet-powder mixtures are installed in the galleries in dry state as a granular assembly. The granular material undergoes hydration by the pore water of the host rock and progressively becomes homogeneous. Before homogenisation, the mechanical behaviour of the material is controlled by its granular nature.

The present work aims at proposing a modelling approach accounting for particular features of pelletpowder mixtures. Two domains are considered: $i$ ) granular, controlled by interactions between pellets with powder in free-swelling conditions in inter-pellet voids, and ii) homogeneous. The behaviour in granular state is studied using Discrete Element Method (DEM). A modified Barcelona Basic Model (BBM) [6] is used to describe the behaviour of the homogenised mixture. Transition from granular to homogeneous states is described by criteria depending on suction and volume fractions of pellets and powder.

First, swelling pressure tests performed in the laboratory on three pellet-powder mixtures are presented. Then, DEM simulations of periodic pellet assemblies are presented. Model equations proposed for granular and homogeneous states are then introduced. Finally, numerical simulations of swelling pressure tests are performed using the proposed model.

\section{Swelling pressure tests}

\subsection{Material}

Three MX80 pellet-powder mixtures are used to perform swelling pressure tests. Pellets are subspherical. Their diameter is $7 \mathrm{~mm}$ and initial suction $\left(s_{0}\right)$ and dry density are $89 \mathrm{MPa}$ and $1.91 \mathrm{Mg} / \mathrm{m}^{3}$ respectively [7]. Powder is made of crushed pellets. The reference mixture has pellet-powder proportions of 70/30. All mixtures have the same mass of pellets but various powder contents. Mixtures are referred to as 70/30, 70/15 and 70/0. Volume fraction of pellets $\left(\Phi_{1}\right)$ and powder $\left(\Phi_{2}\right)$ are defined as the ratio of total volume of pellets/powder to total volume of the sample. Material properties are presented in Table 1.

Table 1. Material used for swelling pressure tests.

\begin{tabular}{|c|c|c|c|}
\hline Mixture & Total dry density, $\rho_{d}$ & $\Phi_{1}$ & $\Phi_{2}$ \\
\hline $70 / 30$ & $1.50 \mathrm{Mg} / \mathrm{m}^{3}$ & 0.553 & 0.237 \\
\hline $70 / 15$ & $1.28 \mathrm{Mg} / \mathrm{m}^{3}$ & 0.553 & 0.118 \\
\hline $70 / 0$ & $1.05 \mathrm{Mg} / \mathrm{m}^{3}$ & 0.553 & 0 \\
\hline
\end{tabular}




\subsection{Methods}

Hydration is performed in vapour phase using the vapour equilibrium technique [8]. Suction $(s)$ values imposed are $82,59,40,38,25,13,9$, and $4 \mathrm{MPa}\left(s_{0}=89 \mathrm{MPa}\right)$. Following the $4 \mathrm{MPa}$ step, hydration is performed by liquid water flooding (same composition as the pore water of the host rock of Bure underground research laboratory). Mixtures are laid in isochoric cylindrical cells. A pressure sensor is placed on the top wall to measure the swelling pressure. Cell diameter is $60 \mathrm{~mm}$, cell height is $30 \mathrm{~mm}$, sensor diameter is $30 \mathrm{~mm}$. One test is performed on 70/30 and 70/15 mixtures, two are performed on 70/0 mixture.

\subsection{Experimental results}

Results are presented in Figure 1. Final swelling pressure of the 70/30, 70/15, and 70/0 materials are 4.45, 0.902, $0.249 \mathrm{MPa}$. These values are close to estimated values associated to their dry density [9], which suggests that all granular mixtures have reached homogenisation.

Upon suction decrease at $s \geq 4 \mathrm{MPa}$, the swelling pressure in 70/30 test (densest powder phase) keeps increasing. In 70/15 and 70/0 test, swelling pressure increases, then reaches a plateau/decreases. No significant differences are observed between the responses of these two tests at $s \geq 4 \mathrm{MPa}$.

It is considered that, owing to the powder density in $70 / 30$ test, the influence of granular structure is not significant. Conversely, powder is considered to be close to free-swelling conditions in 70/15 test. The granular structure controls the macroscopic response at suctions higher than $4 \mathrm{MPa}$. At lower suctions, the material homogenises and the swelling behaviour is not controlled by granular structure.

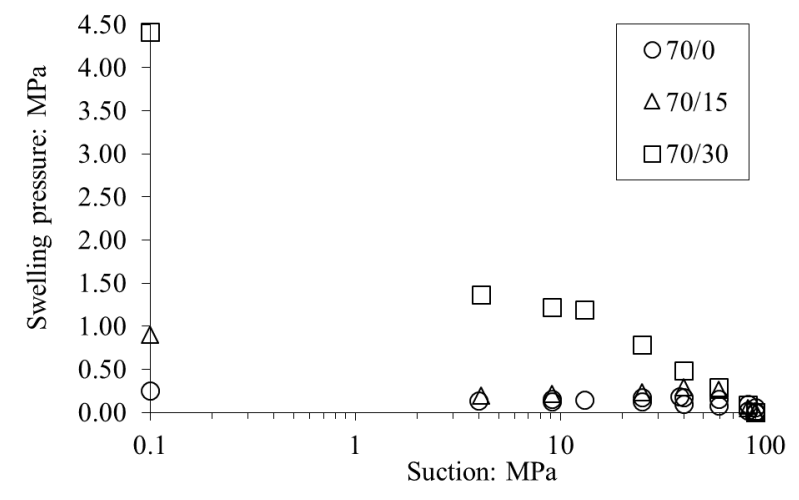

Figure 1. Results of swelling pressure tests.

\section{DEM simulations of pellet assemblies}

\subsection{Method}

\subsubsection{Overview}

DEM simulations of pellet assemblies have been performed in Ref. [10]. It was suggested that the intrinsic behaviour of pellet assemblies is better addressed in large granular assemblies than in simulations of swelling pressure tests. In this respect, it is proposed to determine constitutive laws for pellet assemblies from DEM simulations of large assemblies.

Isotropic compressions of pellet assemblies are performed using DEM. Pellets are modelled as spheres of constant diameter $a=7.53 \mathrm{~mm}$ to keep identical mass and density as real pellets. The same numerical method as in Ref [11] is used. The same numerical samples as in Ref. [10] are used: periodic cubic samples composed of 4000 beads, with varying initial value of $\Phi_{1}\left(\Phi_{l}{ }^{(0)}\right)$. Values of $\Phi_{1}^{(0)}$ considered in this study are: 0.638 ; $0.628 ; 0.595 ; 0.577$. The initial number of coordination in these samples are respectively: $6.0 ; 5.8 ; 4.4 ; 4.4$ [11].

The total volumetric strain of the pellet assemblies $\left(\varepsilon_{V}\right)$ is characterised as a function of a dimensionless pressure parameter $\left(m_{g}\right)$. Contacts are elastic perfectly plastic, using Hertz's law in elasticity and pellet strength as the elastic limit of contacts.

\subsubsection{Dimensionless pressure parameter}

In real pellet assemblies, the macroscopic response upon mechanical loading depends on suction because this latter affects pellet stiffness [7]. To consider this phenomenon, a dimensionless pressure parameter, $m_{g}$, is used in DEM to account for both mean stress and pellet stiffness variations. In this respect, characterising the volumetric behaviour under $m_{g}$ variations allows to determine the material response under both mean stress and pellet stiffness variations.

The mean stress in a granular assembly, $p$, can be written as follows [11, 12]:

$$
p=\frac{1}{3 V} N_{c}<r F>
$$

Where $V$ is the volume of the granular assembly, $N_{c}$ is the number of contacts in the assembly, $<>$ is the mean value for all contacts, $r$ is the distance between two pellets in contact and $F$ is the contact force, given by Hertz's law:

$$
F=\frac{1}{3} \frac{E}{1-v^{2}} a^{\frac{1}{2}} \delta^{\frac{3}{2}}
$$

where $E$ and $v$ are the pellet Young modulus and Poisson ratio, and $\delta$ is the normal deflection at contact between two pellets.

From equations (1) and (2) it appears that volume changes in the granular assembly can be related to the following ratio:

$$
m_{g}=\left(\frac{p}{\frac{E}{1-v^{2}}}\right)^{\frac{2}{3}}
$$

which introduces the aforementioned $m_{g}$. 


\subsubsection{Note concerning pellet swelling}

In real pellet assemblies, particle swelling occurs along with stiffness variation [7]. Since this latter is addressed by $m_{g}$, only the influence of variation of $a$ on $\varepsilon_{V}$ needs to be characterised in the model. It is however not addressed using DEM. Indeed, considering particle swelling (variation of $a$ ) at constant $m_{g}$, assuming constant $N_{c}$ in these conditions, it is easily shown from equations (1), (2), and (3), that:

$$
\varepsilon_{V}=\varepsilon_{V 1}
$$

where $\varepsilon_{V I}$ is the pellet volumetric strain.

\subsection{Hydromechanical behaviour of a pellet}

In DEM, each particle has the same behaviour as a pellet. The model proposed in Ref. [7] is used to describe the pellet behaviour [10]. The main model equations are summarised as follows:

$$
\begin{gathered}
E=3(1-2 v) \frac{1}{\beta_{m}} \exp \left(\alpha_{m} p_{1}^{\prime}\right) \\
\varepsilon_{V 1}=\frac{\beta_{m}}{\alpha_{m}}\left[\exp \left(-\alpha_{m} p_{1}^{\prime}\right)-\exp \left(-\alpha_{m} s_{0}\right)\right] \\
R=C E
\end{gathered}
$$

where $R$ is the elastic limit of the pellet, $p_{l}$ ' is the effective mean stress applied to the pellet, $\alpha_{m}, \beta_{m}$ and $C$ are model parameters [7].

\subsection{Numerical results}

Figure 2 presents the evolution of $\varepsilon_{V}$ as a function of $m_{g}$ in DEM simulations for samples of various $\Phi_{1}{ }^{(0)}$. A bilinear relationship is obtained. The slope change occurs at a threshold value of $m_{g}$, denoted by $m_{g}{ }^{*}$, which appears to depend on $\Phi_{I}{ }^{(0)}$ (Figure 3). Equality $m_{g}=m_{g}{ }^{*}$ corresponds to the occurrence of contact plasticity in the granular assembly.

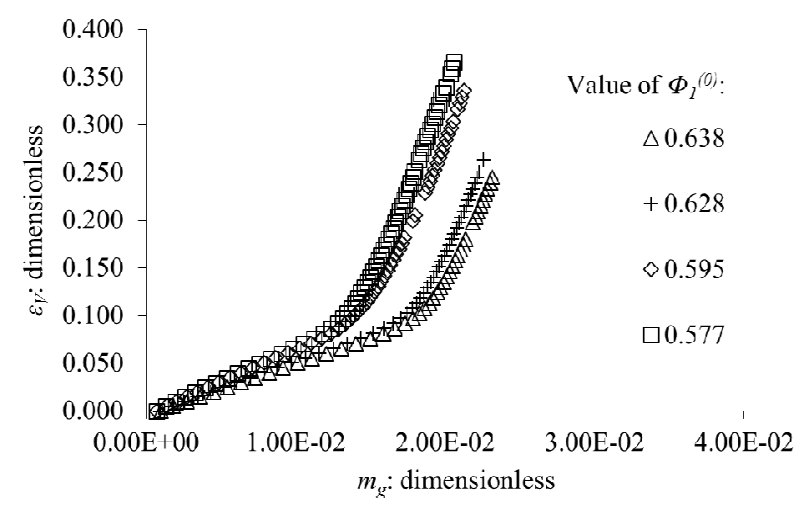

Figure 2. Evolution of $\varepsilon_{V}$ as a function of $m_{g}$ for various $\Phi_{l}{ }^{(0)}$.

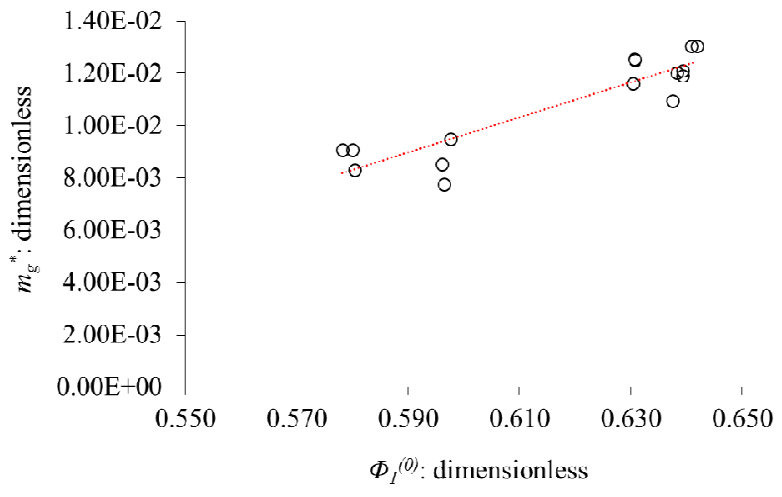

Figure 3. Results of swelling pressure tests.

DEM results are interpreted as follows:

$$
\begin{gathered}
\varepsilon_{V}=f_{\varepsilon a} m_{g} \quad ; \quad m_{g}<m_{g}^{*} \\
\varepsilon_{V}=f_{\varepsilon b} m_{g} ; \quad m_{g} \geq m_{g}^{*} \\
m_{g}^{*}=f_{m a} \Phi_{1}^{(0)}+f_{m b}
\end{gathered}
$$

where $f_{\varepsilon a}, f_{\varepsilon b}, f_{m a}$ and $f_{m b}$ are model parameters (see Table 2).

\section{Model for pellet-powder mixtures}

\subsection{General remark}

The proposed model is based on hardening elastoplasticity. It accounts for two-stress variables, $p$ and $s$. Plasticity and hardening are common to both granular and homogeneous domains. Elastic laws are different depending on the state of the material. The model is described in the following subsections.

\subsection{Granular-homogeneous transition}

In the present model, the main hypothesis is the consideration of two distinct domains. The material is either considered granular or homogeneous. In the granular domain, interactions between pellets control the mechanical behaviour of the assembly and DEM results are used to determine constitutive laws. In the homogeneous domain, the mixture is considered to have homogenised and its behaviour is described using a modified version of the BBM [6]. In non-reactive granular materials, it is considered that coarse particles control the mechanical behaviour if the proportion of small particles is smaller than a threshold value. In this case, it is not relevant for characterising the transition since volumetric proportion of powder remains nearly constant upon hydration.

Experimental results in literature [13] suggest that bentonite materials undergo a significant fabric rearrangement at low suction. As a consequence, it is considered that below a threshold suction, denoted by $s^{*}$, the material behaviour is no longer controlled by the granular structure. 
The main hypothesis associated to the granular domain is that powder is in free-swelling conditions (Figure 1). It is considered that this condition is no longer verified if the volume fraction of powder in interpellet voids $\left(\Phi_{m a t}\right)$ reached a threshold value, denoted by $\Phi_{\text {mat }}{ }^{*}$.

Transition from granular to homogeneous states is considered irreversible. Because of lack of data, parameters $s^{*}$ and $\Phi_{m a t}{ }^{*}$ are estimated. The following values are proposed: $s^{*}=3 \mathrm{MPa}[7,13]$ and $\Phi_{\text {mat }}{ }^{*}=\Phi_{1}$ (i.e. powder in the inter-pellet voids reaches the same volume fraction as pellets in the total volume). Note that it is a first estimation and that pellets are likely not to totally control the macroscopic response until one of these criteria is verified. Conversely, influence of the granular structure is likely not to be immediately totally lost as soon as a criterion is verified.

\subsection{Elastic volumetric strains}

\subsubsection{Granular material}

Elastic volumetric strain $\varepsilon_{V}{ }^{e}$ is considered a function of $m_{g}$ and $\varepsilon_{V l}$. Since both depend on $p$ and $s, \mathrm{~d} \varepsilon_{V}{ }^{e}$ is written as:

$$
\begin{aligned}
& \mathrm{d} \varepsilon_{V}^{e}=\left(\frac{\partial \varepsilon_{V}^{e}}{\partial m_{g}} \frac{\partial m_{g}}{\partial p}+\frac{\partial \varepsilon_{V}^{e}}{\partial \varepsilon_{V 1}} \frac{\partial \varepsilon_{V 1}}{\partial p}\right) \mathrm{d} p \\
& +\left(\frac{\partial \varepsilon_{V}^{e}}{\partial m_{g}} \frac{\partial m_{g}}{\partial s}+\frac{\partial \varepsilon_{V}^{e}}{\partial \varepsilon_{V 1}} \frac{\partial \varepsilon_{V 1}}{\partial s}\right) \mathrm{d} s
\end{aligned}
$$

where, from equation (8), $\frac{\partial \varepsilon_{V}^{e}}{\partial m_{g}}=f_{\varepsilon a}$ or $f_{\varepsilon b}$, and $\frac{\partial \varepsilon_{V}^{e}}{\partial \varepsilon_{V 1}}=1$.

According to hypotheses discussed in Ref. [7], pellets, and in this case powder grains, are considered elastic, composed of micropores [14] only, and fullysaturated. In the granular domain, $\varepsilon_{V I}$ is a function of $p$ and $s$, and powder volumetric strain, $\varepsilon_{V 2}$, is only a function of $s$ :

$$
\mathrm{d} \varepsilon_{V 1}=\beta_{m} \exp \left(-\alpha_{m} p_{1}^{\prime}\right) \mathrm{d} p_{1}^{\prime}
$$

where, in the granular domain,

$$
p_{1}^{\prime}=\frac{p}{\Phi_{1}}+s
$$

And

$$
\mathrm{d} \varepsilon_{V 2}=\beta_{m} \exp \left(-\alpha_{m} s\right) \mathrm{d} s
$$

Values of model parameters are summarised in Table 2.

\subsubsection{Homogenised material}

The initial BBM [6] is adapted to account for more than one porosity. Three void ratios are considered: i) macrostructural void ratio $\left(e_{M}\right)$, associated to the volume of voids between pellets and powder grains $\left(\Omega_{v M}\right)$, ii) pellet and iii) powder grain microstructural void ratios $\left(e_{m 1}\right.$ and $\left.e_{m 2}\right)$, associated to intra-pellet and intra-powder grain volume of voids $\left(\Omega_{v 1}\right.$ and $\left.\Omega_{v 2}\right)$. Respectively denoting by $\Omega_{s 1}$ and $\Omega_{s 2}$ the volume of solid in pellets and powder, void ratios are defined as:

$$
\begin{gathered}
e_{M}=\frac{\Omega_{v M}}{\Omega_{s 1}+\Omega_{s 2}} \\
e_{m 1}=\frac{\Omega_{v 1}}{\Omega_{s 1}} \\
e_{m 2}=\frac{\Omega_{v 2}}{\Omega_{s 2}}
\end{gathered}
$$

Total void ratio, $e$, is written as:

$$
e=e_{M}+\frac{\Omega_{s 1}}{\Omega_{s 1}+\Omega_{s 2}} e_{m 1}+\frac{\Omega_{s 2}}{\Omega_{s 1}+\Omega_{s 2}} e_{m 2}
$$

Dimensionless stiffness parameters for the macrostructure, $\kappa$ and $\kappa_{s}$, and for the microstructure, $\kappa_{m}$, describe the elastic (superscript $e$ ) increment of void ratios of the different levels of structure as functions of the stress variables of the model:

$$
\begin{gathered}
\mathrm{d} e_{M}^{e}=-\left(\kappa \frac{\mathrm{d} p}{p}+\kappa_{s} \frac{\mathrm{d} s}{s+p_{a t m}}\right) \\
\mathrm{d} e_{m 1}=-\kappa_{m} \frac{\mathrm{d} p_{1}^{\prime}}{p_{1}^{\prime}} \\
\mathrm{d} e_{m 2}=-\kappa_{m} \frac{\mathrm{d} p_{2}^{\prime}}{p_{2}^{\prime}}
\end{gathered}
$$

where $p_{a t m}$ is the atmospheric pressure and $p_{2}{ }^{\prime}$ is the powder effective mean stress.

In the present work, hydraulic equilibrium is assumed between each level of structure for simplicity. In the homogeneous domain, powder is assumed to be as affected as pellets by mechanical loadings. Effective stresses applied to both pellets and powder are thus identical. Thus, $p_{1}{ }^{\prime}=p_{2}$ ' and $\mathrm{d} e_{m 1}=\mathrm{d} e_{m 2}$.

Consequently, from equation (17), denoting by $e_{0}$ the initial void ratio:

$$
\mathrm{d} \varepsilon_{V}^{e}=\frac{1}{1+e_{0}}\left(\mathrm{~d} e_{M}^{e}+\mathrm{d} e_{m}\right)
$$

With

$$
e_{m}=\frac{\Omega_{s 1}}{\Omega_{s 1}+\Omega_{s 2}} e_{m 1}+\frac{\Omega_{s 2}}{\Omega_{s 1}+\Omega_{s 2}} e_{m 2}
$$

And

$$
\mathrm{d} e_{m}=\mathrm{d} e_{m 1}=\mathrm{d} e_{m 2}=-\kappa_{m} \frac{\mathrm{d} p_{1}^{\prime}}{p_{1}^{\prime}}
$$

Where, in the homogeneous domain, 


$$
p_{1}^{\prime}=\frac{p}{\Phi_{1}+\Phi_{2}}+s
$$

\subsection{Plasticity and hardening}

Plasticity and hardening are addressed as in the original BBM [6]. The most important equations are summarised hereafter. For further details, interested readers may directly refer to Ref. [6].

The yield surface of the model is described as:

$$
q^{2}-M^{2}\left(p+k_{s} s\right)\left(p_{0}(s)-p\right)=0
$$

with $q$ the deviatoric stress, $M$ the slope of the critical state line, $p_{0}$ the preconsolidation stress, function of $s$ :

$$
p_{0}(s)=p_{c}\left(\frac{p_{0}(0)}{p_{c}}\right)^{\frac{\lambda(0)-\kappa}{\lambda(s)-\kappa}}
$$

With $p_{c}$ a reference stress and $\lambda$ the elasto-plastic dimensionless stiffness parameter, function of $s$ :

$$
\lambda(s)=\lambda(0)\left[r+(1-r) \exp \left(-\beta_{M} s\right)\right]
$$

where $\lambda(0), r$, and $\beta_{M}$ are model parameters.

Hardening law is written:

$$
\frac{\mathrm{d} p(0)}{p(0)}=\frac{1+e_{0}}{\lambda(0)-\kappa} \mathrm{d} \varepsilon_{V}^{p}
$$

where $\mathrm{d} \varepsilon_{V}{ }^{p}$ is the increment of total plastic volumetric strain, calculated from the consistency condition accounting for an associated flow rule. Note that, since microstructure is considered elastic, $\mathrm{d} \varepsilon_{V}^{p}$ only affects $e_{M}$.

\section{Simulations of laboratory tests}

\subsection{Method}

The model is used to simulate swelling pressure tests. $s$ is decreased from $s=s_{0}$ to $s=0.1 \mathrm{MPa}$. Gas pressure is taken equal to 0 for simplicity. Gravity is not considered. No volumetric strain is allowed.

\subsection{Model parameters}

Parameters related to the granular domain are obtained from Ref. [7] or from DEM simulation results. Parameters related to the transition from granular to homogeneous states are estimated as described in 4.2. Parameters related to the homogenised domain are conveniently estimated.

In bentonite materials, the swelling potential and apparent preconsolidation stress increase with increasing dry density. To account for these features, it is proposed to estimate $p_{0}(0)$ from results of oedometer tests performed on MX80 materials, available in Ref. [15-17] (Figure 4). The following relationship is proposed:

$$
p_{0}(0)=p_{0}^{*} \exp \left(-n_{p} e_{0}\right)
$$

where $p_{0}{ }^{*}$ and $n_{p}$ are parameters deduced from experimental results.

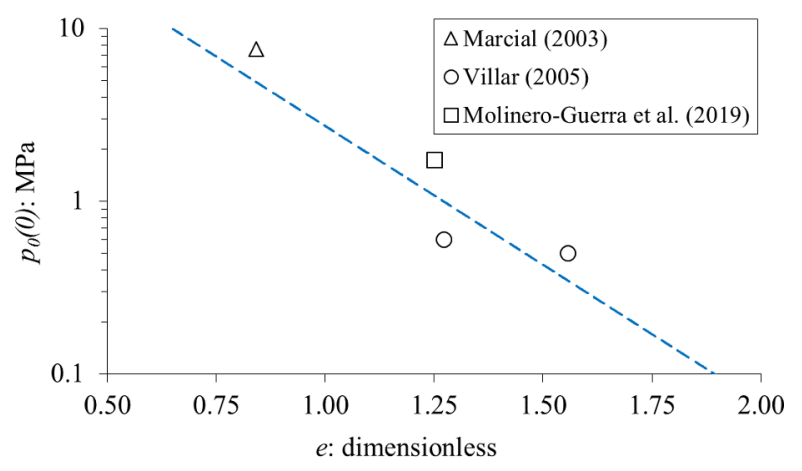

Figure 4. Saturated preconsolidation stress as a function of void ratio for MX80 materials. Results from Marcial [15], Villar [16], and Molinero-Guerra et al. [17]. Dashed line represents equation (29) for $p_{0}{ }^{*}=80 \mathrm{MPa}$ and $n_{p}=3.7$

To allow the swelling potential to increase with increasing dry density while avoiding to fit an additional parameter, it is proposed to allow $\kappa_{s}$ to vary from 0 to $\kappa_{m}$ according to the following relationship:

$$
\kappa_{s}=\frac{e_{m 0}}{e_{0}} \kappa_{m}
$$

where $e_{m 0}$ is the initial value of $e_{m}$. In this respect, in dense materials, as $e_{0}$ approaches $e_{m 0}, \kappa_{s}$ would approach $\kappa_{m}$ and a higher swelling potential would be computed. This approach is not directly supported by experimental evidence. It is suggested that other methods allowing $\kappa_{s}$ to increase with increasing $\rho_{d}$ could be suitable. $\kappa_{m}$ and $\kappa$ are thus the only elastic stiffness parameters to estimate in the homogenised domain.

The three studied mixtures have the same values of $\Phi_{1}^{(0)}=0.553$ and $e_{m 0}=0.45 . e_{0}$ is respectively equal to $1.64,1.17,0.847$ for the $70 / 0,70 / 15$, and $70 / 30$ materials.

\subsection{Results and discussion}

Using a single set of parameters (Table 2) and initial material properties (Table 1), the three swelling pressure tests are simulated. Results are compared to experiments in Figure 5.

Although some differences between experimental and model results are obtained, the main trends of the swelling pressure evolution are satisfactorily reproduced. In particular, the homogenisation of the 70/30 material at high suction, the response of the 70/15 material controlled by the pellet assembly until low suction, and final swelling pressures for all materials are close to experiments. 
Table 2. Model parameters.

\begin{tabular}{|c|c|}
\hline Notation & Value \\
\hline \multicolumn{2}{|c|}{ Granular domain } \\
\hline$\alpha_{m}$ & $0.024 \mathrm{MPa}^{-1}$ \\
\hline$\beta_{m}$ & $0.016 \mathrm{MPa}^{-1}$ \\
\hline$f_{\varepsilon a}$ & 6 \\
\hline$f_{\varepsilon b}$ & 40 \\
\hline$f_{m a}$ & 0.067 \\
\hline$f_{m b}$ & -0.031 \\
\hline \multicolumn{2}{|c|}{ Transition } \\
\hline$s^{*}$ & $3 \mathrm{MPa}$ \\
\hline$\Phi_{m a t}{ }^{*}$ & $=\Phi_{1}$ \\
\hline \multicolumn{2}{|c|}{ Homogeneous domain } \\
\hline$\kappa$ & 0.025 \\
\hline$\kappa_{m}$ & 0.015 \\
\hline \multicolumn{2}{|c|}{ Plasticity and hardening } \\
\hline$\lambda(0)$ & 0.20 \\
\hline$p_{c}$ & $0.050 \mathrm{MPa}$ \\
\hline$r$ & 0.8 \\
\hline$\beta_{M}$ & $0.1 \mathrm{MPa}^{-1}$ \\
\hline$p_{0}{ }^{*}$ & $80 \mathrm{MPa}$ \\
\hline$n_{p}$ & 3.7 \\
\hline
\end{tabular}

Some hypotheses have been considered in the estimation of parameters. Hydraulic equilibrium between pellets and powder can induce a lower suction in pellets in the model compared to experiment, and thus a computed homogenisation at higher imposed $s$. Parameters in the granular domain are constant in the model whereas, from Figure 2, a dependency of $f_{\varepsilon a}$ and $f_{\varepsilon b}$ on $\Phi_{l}^{(0)}$ may seem appropriate. Also, it is considered that $\frac{\partial \varepsilon_{V}^{e}}{\partial \varepsilon_{V 1}}=1$, which is exact only if all contacts are elastic. The computed response can thus be characterised by a higher apparent stiffness in the granular domain. Dimensionless stiffness parameters in the homogenised domain are also considered constant, and their values are conveniently estimated without direct experimental evidences. It is however worth mentioning that $\kappa, \lambda(0), r$ and $\beta_{M}$ are close to values proposed in Ref. [5] to model pellet-powder mixtures. Finally, a more accurate description of the transition from granular to homogeneous states is an interesting perspective of the present work.

In spite of these simplifications, the conceptual approach proposed in the present work appears to provide interesting results regarding the modelling of pellet-powder mixtures, where powder can be in freeswelling conditions in granular state and the material can homogenise upon hydration.

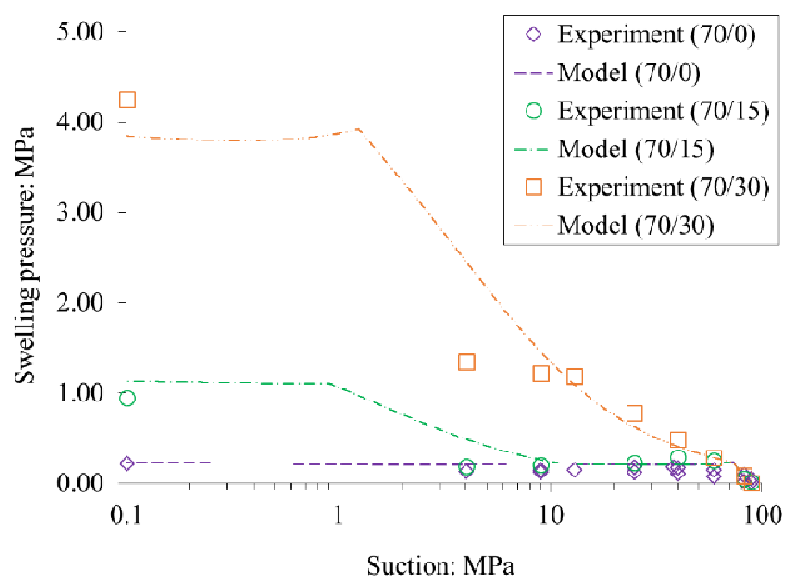

Figure 5. Comparison of experimental and model results for the three swelling pressure tests, using a single set of parameters.

\section{Conclusion}

A conceptual approach was proposed to model the hydromechanical behaviour of bentonite pellet-powder mixtures. Constitutive laws and parameters related to the granular state are obtained through DEM simulations. The behaviour of the homogeneous material is described by a modified version of the BBM.

The proposed approach is able to address particular features of these materials such as powder in freeswelling conditions, mechanical behaviour controlled by pellet assemblies in loose-powder materials, transition to homogeneous state, increase of swelling potential and preconsolidation stress with increasing dry density.

The material behaviour is simplified and improvement of the approach can be proposed in future works. However, in spite of these simplifications, three swelling pressure tests performed in the laboratory on pellet-powder mixtures with various powder contents were satisfactorily simulated using a single set of parameters, which none of the traditional modelling framework based only on a continuum approach can achieve.

\section{References}

1. van Geet, M., Volckaert, G., Roels, S., Applied Clay Science, 29 (2005)

2. Imbert, C., Villar, M. V., Applied Clay Science, 32 (2006)

3. Hoffmann, C., Alonso, E. E., Romero, E., Physics and Chemistry of the Earth, 32 (2007)

4. Gens, A., Valleján, B., Sánchez, M., Imbert, C., Villar, M. V., van Geet, M., Géotechnique, 61(5) (2011)

5. Mokni, N., Molinero-Guerra, A., Cui, Y.-J., Delage, P., Aimedieu, P., Bornert, M., Tang, A. M., Géotechnique, (2019)

6. Alonso, E. E., Gens, A., Josa, A., Géotechnique, 40(3) (1990) 
7. Darde, B., Tang, A. M., Pereira, J.-M., Roux, J.-N., Dangla, P., Talandier, J., Vu, M. N., Géotechnique Letters, 8(4) (2018)

8. Tang, A.-M., Cui, Y.-J., Canadian Geotechnical Journal, 42(1) (2005)

9. Wang, Q., Tang, A. M., Cui, Y. J., Delage, P., Gatmiri, B., Engineering Geology, 124(1) (2012)

10. Darde, B., Roux, J. N., Dangla, P., Pereira, J. M., Tang, A. M., Talandier, J., Vu, M. N, in CIGOS 2019, (2020)

11. Agnolin, I., Roux, J. N., Physical Review E, 76(6) (2007).

12. Christoffersen, J., Mehrabadi, M. M., NematNasser, S., Journal of Applied Mechanics, 48(2) (1981)

13. Saiyouri, N., Tessier, D., Hicher, P. Y, Clay Minerals, 39(4) (2004)

14. Alonso, E. E., Vaunat, J., Gens, A., Engineering Geology, 54(1-2) (1999)

15. Marcial, D., Doctoral dissertation, École nationale des ponts et chaussées (2003). In French.

16. Villar, M. V., Informes Técnicos Ciemat, $\mathbf{1 0 5 3}$ (2005)

17. Molinero-Guerra, A., Cui, Y. J., He, Y., Delage, P., Mokni, N., Tang, A. M., Aimedieu, P., Bornert, M., Bernier, F. Engineering Geology, 248 (2019) 